\title{
Software Security Vulnerability
}

National Cancer Institute

\section{Source}

National Cancer Institute. Software Security Vulnerability. NCI Thesaurus. Code C92116.

The device software failed to provide adequate authorization, access control, protection and accountability features. 